MMCA-97 Conference, pp. 117-121

R. Čiegis (Ed)

(C) 1997 Vilniaus Gedimino technikos universitetas

\title{
ON THE USE OF THE CLASSICAL NUMERICAL METHODS FOR DIFFERENTIAL DELAY EQUATIONS
}

\author{
MEČISLOVAS MEILŪNAS and RŪTA RUMŠIENE் \\ Vilnius Gediminas Technical University \\ Sauletekio ave. 11, LT-2054 Vilnius \\ Klaipeda University \\ H. Manto ave. 84, Klaipèda
}

\section{ABSTRACT}

An algorithm for the evaluatinion of discontinuity jumps in the DDE initial value problem is presented. That enables the use of the classical numerical methods for DDE initial value problem.

\section{INTRODUCTION}

The need for numerical solution of initial value problem for differential delay equations (DDE) arises from the fact that many biological phenomena are modelled by nonlinear equations of this type. Logistic equation [1]

$$
\dot{x}=r x\left(1-\frac{x_{\tau}}{K}\right)
$$

and infectional desease model, proposed by G.I.Marchuk [2]

$$
\begin{aligned}
\dot{v} & =\left(h_{1}-h_{2} f\right) v \\
\dot{f} & =h_{4}(s-f)-h_{8} f \cdot v \\
\dot{\xi} & =h_{3} \xi(m) \cdot f_{\tau} \cdot v_{\tau} \cdot \Theta_{\tau}-h_{5}(s-1) \\
\dot{m} & =h_{6} v-h_{7} m
\end{aligned}
$$

where $\dot{\Phi}(t)=\frac{d \Phi(t+0)}{d t}, \Phi_{\tau}=\Phi(t-\tau), \tau$ - time delay constant, $\Theta(t)$ - Heaviside function, $r, K, h_{i}, i=1, \ldots, 8$ - model constants, are well known examples of such models. 
It is one essential distinction between ODE and DDE: the solution of DDE initial value problem is non-smooth [3]. We will ilustrate this basic property of DDE with very simple example.

Example. Consider a initial value problem

$$
\begin{aligned}
\dot{x} & =x_{\tau}, \quad \tau=1, \\
\dot{x}(t) & =1, \quad \text { if } \quad-\tau \leq t \leq 0,
\end{aligned}
$$

where $\dot{x}$ is the right derivative of $x(t)$.

The solution can be expressed in explicit form by using method of steps [3]. It is convienient to set $x(0)=\varphi(0)$. Thus the solution is

$$
x(t)=\left\{\begin{array}{lr}
1, & -1 \leq t<0, \\
t+1, & 0 \leq t<1, \\
\frac{t^{2}}{2}+\frac{3}{2}, & 1 \leq t<2, \\
\frac{t^{3}}{6}-\frac{t^{2}}{2}+2 t+\frac{1}{6} & 2 \leq t<3,
\end{array}\right.
$$

wherefrom we obtain

$$
\begin{aligned}
& \Delta x_{0}^{(0)} \stackrel{\text { def }}{=} x(+0)-x(-0)=0 \\
& \Delta x_{0}^{(1)} \stackrel{\text { def }}{=} \dot{x}(+0)-\dot{x}(-0)=1 \\
& \Delta x_{0}^{(2)} \stackrel{\text { def }}{=} \ddot{x}(+0)-\ddot{x}(-0)=1 \\
& \Delta x_{0}^{(3)} \stackrel{\text { def } \dddot{x}}{=} x(+0)-\dddot{x}(-0)=2 \\
& \quad \ldots \ldots \ldots \ldots \ldots \ldots
\end{aligned}
$$

Therefore the use of classical numerical methods for DDE is troublesome. It has been shown in [3], that Runge-Kutta and Adams methods of high accuracy do not work perfectly in case (3), (4).

\section{THE SOLUTION DISCONTINUITY PROBLEM IN NUMER- ICAL METHODS}

More generally, the $k$-th derivative of the initial value problem

$$
\begin{aligned}
& \dot{x}(t)=f\left(x(t), x_{\tau}(t), t\right) \\
& x(t)=\varphi(t) \quad \text { for } \quad-\tau \leq t \leq 0
\end{aligned}
$$

solution has finite discontinuity at point $t_{k}=(k-1) \tau$ even for smooth $f(u, v, w)$. Thus, the DDE solution has smoothing property: for $t>k \tau$ its $k$-th derivative is continuous. Therefore, the high order accuracy numerical schemes are inapplicable in the initial part of integration interval only. 
There are several ways to overcome this difficulty:

- to choose small integration step $h$ in this initial part and any simple integration method (e.g. Euler scheme),

- to choose the integration step $h=\frac{\tau}{p}$, where $p$ is positive integer and to use right derivative of the solution at its discontinuity point,

- to evaluate the derivatives discontinuity jumps in order to use this information in numerical schemes construction [4].

Consider the use of the evaluation of $x^{(j)}(t)$ jumps at $t=(j-1) t$ in order to solve any equivalent problem with smooth solution (cf.[4]).

The following theorem is basic for further considerations.

THEOREM [4]. Let $f(t), t \in(a, b)$ be a piecewise continuous function, which $j$-th derivatives, $j=0,1, \ldots, \nu$ are continuous in intervals $\left(t_{k-1}, t_{k}\right), a \leq t_{1}<$ $t_{2}<\ldots<t_{N} \leq b$, and have first kind discontinuity at points $t=t_{k}$. Denote $\Delta f_{k}^{(j)}, j=0, \ldots, \nu, k=1, \ldots, N$ the discontinuity jump of the $j$-th derivative at $t=t_{k}$. There exist a smoothing function $g(t)$, for which the difference $u(t)=f(t)-g(t)$ is $(\nu-1)-$ differentiable in interval $(a, b)$. The function $g(t)$ can be expressed as follows:

$$
g(t)=\sum_{k=1}^{N} \Theta\left(t-t_{k}\right) \sum_{j=0}^{\nu-1} f_{k}^{(j)} \frac{\left(t-t_{k}\right)^{j}}{j !},
$$

where

$$
\Theta\left(t-t_{k}\right)= \begin{cases}0, & t<t_{k} \\ 1, & t \geq t_{k}\end{cases}
$$

Let us consider the initial value problem (5), (6). $j$-th order derivatives of its solution have first kind discontinuity jumps $\Delta x_{k}^{(j)}$ at points $t_{k}=k \tau$. Evaluation of $\Delta x_{k}^{(j)}(j=1, \ldots, L+1, k=0, \ldots, L)$ enables to construct the function $u(t)=x(t)-g(t)$, which is $(L+1)$ - differentiable and satisfies the following equation:

$$
\dot{u}=f\left(x+g, x_{\tau}+g_{\tau}, t\right)-g^{\prime}(t) \stackrel{\text { def }}{=} W\left(t, u, u_{\tau}\right)
$$

and initial condition

$$
u(t)=\varphi(t)
$$

where

$$
g^{\prime}(t)=\sum_{k} \sum_{j=1}^{L+1} \Delta x_{k}(j) \frac{\left(t-t_{k}\right)^{j-1}}{(j-1) !} \Theta\left(t-t_{k}\right)
$$

Thus now we have a initial value problem (7), (8) with smooth solution, for which one can apply classical numeral methods. 


\section{EVALUATION OF DISCONTINUITY JUMPS}

If the right-hand side of (4) is sufficiently smooth function of its arguments, we can evaluate $\Delta_{k}^{(j)}$ in most elementary way, then proposed in [4]. In order to apply any classical method for the problem (5), (6) we may evaluate the quantities

$$
\begin{aligned}
& \Delta x_{0}^{(1)} \\
& \Delta x_{0}^{(2)}, \Delta x_{1}^{(2)} \\
& \ldots \ldots \ldots \ldots \ldots \ldots \ldots \ldots \\
& \Delta x_{0}^{(L+1)}, \Delta x_{1}^{(L+1)}, \ldots, \Delta x_{L+1}^{(L+1)}
\end{aligned}
$$

Let us consider the point $t=t_{0}$. (The values $\Delta x_{k}^{(j)}, k>0$ can be find in similar way, if the solution $x(t)$ is known in interval $[\tau(k-1), \tau k)$.

For $t \in[0, \tau)$ we have

$$
\dot{u}=f\left(u+\sum_{j=1}^{L+1} \Delta x_{0}^{(j)} \frac{t^{j}}{j !}, \varphi(t-\tau), t\right)-\sum_{j=1}^{L+1} \Delta x_{0}^{(j)} \frac{t^{j-1}}{(j-1) !}
$$

$u(t)=\varphi(t)$, when $t \in[-\tau, 0)$.

Denote $\partial_{t}^{j} \varphi_{0}=\varphi^{(j)}(0-0), \partial_{t}^{j} \varphi_{\tau}=\varphi^{(j)}(-\tau+0), f_{1}\left(x, x_{\tau}, t\right)=f\left(x, x_{\tau}, t\right)$, $D^{j} x(t)=\frac{d^{j} x(t)}{d t^{j}}$.

Because of the smoothness we have

$$
\dot{u}(0+0)=\dot{u}(0-0)=\varphi_{0}^{(1)},
$$

for $t \rightarrow 0$ we obtain

$$
\Delta x_{0}^{(1)}=f_{1}\left(\varphi_{0}, \varphi_{\tau}, 0\right)-\varphi_{0}^{(1)} .
$$

Let us differtiate both sides of (5) equation according to $t$ :

$$
\begin{aligned}
D^{2} x= & \partial_{x} f_{1} \cdot f_{1}+\partial_{x_{\tau}} f_{1} \cdot \partial_{t} \varphi_{\tau}+\partial_{t} f_{1}=f_{2}\left(x, \varphi_{\tau}, \partial_{t} \varphi_{\tau}, t\right) \\
D^{3} x= & \partial_{x} f_{2} \cdot f_{2}+\partial_{x_{\tau}} f_{2} \cdot \partial_{t} \varphi_{\tau}+\partial_{t} f_{2} \\
& \ldots \ldots \ldots \ldots \cdots \cdots \cdots \\
D^{j} x= & \partial_{x} f_{j-1} \cdot f_{j-1}+\partial_{x_{\tau}} f_{j-1} \cdot \partial_{t} \varphi_{\tau}+\partial_{t} f_{j-1} \\
= & f_{j}\left(\varphi_{0}, \varphi_{\tau}, \ldots, \partial_{t} \varphi_{\tau}, \ldots, \partial_{t}^{(j-1)} \varphi_{\tau}, 0\right)
\end{aligned}
$$

Therefore we obtain general formula for the jumps at the point $t=0$ :

$$
\Delta x_{0}^{(j)}=\left[f_{j}\left(\varphi_{0}, \varphi_{\tau}, \ldots, \partial_{t}^{(j-1)} \varphi_{\tau}, 0\right)-\partial_{t}^{j} \varphi_{0}\right](j-1) !
$$


Denote $x^{k}=x\left(t_{k}-0\right), x_{\tau}^{k}=x\left(t_{k-1}+0\right), k=0,1, \ldots$ and formulate the general statement about jumps at the points $t=t_{k}$.

THEOREM 2. If $f(u, v, w)$ is smooth enough, then the jumps of the derivattives of the (4), (5) solution $x(t)$ can be evaluated consequently at the points $t_{0}, t_{1}, \ldots, t_{k}, k=0,1$ and expressed as follows:

$$
\Delta x_{k}^{(j)}=\left[f_{j}\left(x^{k}, x_{\tau}^{k}, \partial_{t} x_{\tau}^{k}, \ldots, \partial_{t}^{j-1} x_{\tau}^{k}, t_{k}\right)-\partial_{t}^{j} x^{k}\right](j-1) !
$$

where $f_{j}$ are constructed according to (11).

Corollary. Let $f(u, v, w)$ is smooth function of its arguments. Consider the initial value problem (6), (7). When the initial function $\varphi(t)$ satisfies the following equalities:

$$
f_{j}\left(\varphi_{0}, \varphi_{\tau}, \partial_{t} \varphi_{\tau}, \ldots, \partial_{t}^{(j-1)} \varphi_{\tau}, 0\right)=\partial_{t}^{j} \varphi_{0}, \quad j=1, \ldots, N,
$$

then the solution $x(t)$ has continuous derivatives up to $N$-th order.

\section{REFERENCES}

[1] J.D. Murray. Mathematical Biology. Springer-Verlag. 1990.

[2] Marchuk G.I. Mathematical models in immunology. M.: Nauka, 1985. (In Russian).

[3] Elsgolc L.E, Norkin S.B. Introduction in the theory of differential equations with deviating argument. M.: Nauka, 1971. (In Russian).

[4] Belykh L.N. Analysis of the mathematical models in immunology. M.: Nauka, 1988. (In Russian). 